\title{
La Unión Europea: una receta para el nacionalismo
}

Miguel Martínez

\section{RESUMEN}

Los nacionalismos en Europa que han surgido (o resurgido), sobre todo, a partir de la crisis que comenzó la década anterior, han puesto en punto de mira el proceso de integración europeo. Todos tienen en común la idea de menos Europa para afrontar los problemas como la inmigración, la salida de la crisis, la movilidad del mercado interior..., etc. Lo que se plantea en este artículo es que la UE posee las herramientas para afrontar los retos a los que se enfrentan sus Estados miembros y que se ha venido demostrando que es el plano supranacional el que puede aportar las mejores soluciones.

Palabras clave: nacionalismo, Unión Europea, identidad, instituciones europeas, integración.

\section{The European Union: a recipe for nationalism}

\begin{abstract}
The nationalisms in Europe that have emerged (or resurged), especially since the crisis that began the previous decade, have put the European integration process in focus. All have in common the idea of less Europe in order to deal with problems such as immigration, the way out of the crisis, the mobility of the internal market ... etc. What is proposed in this article is that the EU has the tools to face the challenges faced by its member states and that it has been demonstrated that it is the supranational level that can provide the best solutions.
\end{abstract}

Key words: nationalism, European Union, identity, european institutions, integration.

* Doctor en ciencia política y relaciones internacionales. Docente investigador Cipe, Facultad de Finanzas, Gobierno y Relaciones Internacionales, Universidad Externado de Colombia, Bogotá (Colombia) [miguel.martinezg@ uexternado.edu.co].

Recibido: 8 de mayo de 2018 / Modificado: 1 de agosto de 2018 / Aceptado: 8 de agosto de 2018

Para citar este artículo:

Martínez, M. (2018). La Unión Europea: una receta para el nacionalismo, OASIS, 28, pp. 83-96.

DoI: https://doi.org/10.18601/16577558.n28.06 


\section{INTRODUCCIÓN}

Con el inicio de las Comunidades Europeas y la idea de Jean Monnet de crear un espacio común de paz se pretendía, entre otras cosas, acabar con la posibilidad de que renacieran los nacionalismos que habían llevado a Europa al peor desastre de la humanidad, la segunda guerra mundial. Si se echa un vistazo a la historia de la Unión Europea, sin duda, esta ha resultado un éxito en este sentido, aparte de la ya conocida idea de Charles de Gaulle de la Europa de las naciones, pero nunca en contra de las Comunidades Europeas, no se han producido movimientos nacionalistas significativos en la Unión hasta finales de la década de los noventa cuando surgió como partido que ayudaría a gobernar a la derecha austriaca Jorg Haider ${ }^{1}$. También en Francia, en la segunda legislatura de Jacques Chirac, surgió el Frente Nacional de Jean Marie Le Pen que llegó a enfrentarse al que sería presidente en segunda vuelta, ganando por una cifra histórica del $82 \%$ de los votos.

La paz ya es una constante en la Unión Europea y nadie, en la actualidad, concibe un enfrentamiento entre los Estados europeos, mucho menos después de lo que sucedió en los Balcanes en la década de los noventa. Esto ha provocado que la paz ya no sea un objeti- vo tan visible y la gran cantidad de retos a los que se enfrenta la UE, y sus Estados miembros, está haciendo que surjan movimientos nacionalistas que, aprovechando la situación económica, las oleadas de refugiados de los conflictos colindantes a Europa, los atentados terroristas que se han sucedido en diferentes países de la Unión, etc., piensan que la solución se encuentra dentro de cada Estado y no en la Unión Europea.

El ejemplo más significativo ha sido el del Reino Unido que, tras un referéndum convocado para que los ciudadanos decidieran si permanecer o salirse de la UE, resolvieron que la opción era salirse creyendo todo aquello que los euroescépticos del UKIP les contaron, sobre todo que el Reino Unido ya no decidía nada en su Parlamento, sino que todo se decidía en las instituciones europeas y el Reino Unido no podía permitir que eso sucediera porque iba en contra del mantenimiento de su soberanía ${ }^{2}$. Así, los ingleses optaron por afrontar los retos que son los mismos a los que se enfrentan los demás Estados de la UE, con independencia y siendo un país tercero, lo cual aún no está tan claro ya que las negociaciones para su salida están bastante estancadas y ni siquiera se sabe cómo va a producirse el período transitorio de dos años hasta su salida definitiva ${ }^{3}$.

1 Este líder del Partido Liberal de la extrema derecha austriaca, más adelante mesuró su ideología y creó un partido de carácter más moderado.

2 Dos de los puntos más destacados de la agenda del UKIP fueron los de la soberanía y la inmigración. Para más información ver: https://www.cidob.org/ca/articulos/cidob_report/n1_1/marcando_la_agenda_del_brexit_el_ populismo_y_el_ukip_en_el_reino_unido

3 Hasta la fecha se han producido dos rondas de negociación en las que no se han llegado a acuerdos concretos y ni siquiera se han comenzado a discutir los temas más polémicos sobre la libre circulación de los factores productivos. De hecho, se dice que ya se está hablando de un posible Bregret. 
De manera paralela a lo sucedido en el Reino Unido, se ha producido el ascenso de partidos nacionalistas, tanto de derecha como de izquierda, en otros Estados de la Unión. Cabe destacar el ascenso de Marine Le Pen en Francia ${ }^{4}$, que logró aún más votos que su padre, el ascenso del Partido por la Libertad de Geert Wilders en Holanda, el Partido Fidesz del reelegido presidente de Hungría Viktor Orbán, el Partido Ley y Justicia liderado por Andrzej Duda que gobierna en Polonia ${ }^{5}$, el movimiento de Beppe Grillo en Italia o la Liga Norte, el ascenso de la derecha más conservadora en Dinamarca ${ }^{6}$, el ya nombrado UKIP $^{7}$, en el Reino Unido.

Todos estos partidos políticos, que representan a sus países en el Consejo, en caso de gobernar en sus Estados, o representan a sus votantes en el Parlamento europeo ${ }^{8}$, a pesar de tener ideologías completamente diferentes y de corte más moderado o radical, tienen un objetivo común, hacer desaparecer la Unión Europea y devolver a sus respectivos Estados las competencias que estos han atribuido a la UE, incluso algunos han propuesto regresar a la moneda que tenían antes de entrar en vigor el euro.

Esto quiere decir que estos movimientos son contrarios a la integración cada vez más profunda de la UE y piensan que los retos a los que se enfrentan en la actualidad se pueden resolver a nivel nacional y no a nivel supranacional. De ahí que las propuestas de la Comisión Europea, como aquella de las cuotas de refugiados por Estados miembros, no hayan podido llevarse a cabo, o una mayor cooperación o, por qué no, integración de servicios de inteligencia, no sea una opción aun en el marco de la UE.

Visto de esta manera, que lo contemplan estos movimientos nacionalistas, cabría pensar que todos los retos que enfrentan la UE y los Estados miembros han sido causados por esta, y que la solución se encuentra dentro de cada una de las fronteras nacionales. Han achacado

$4 \quad$ Hija de Jean Marie Le Pen, líder y creador del Frente Nacional. Su hija le ha bajado el tono xenófobo al partido de derecha radical francés para acoger más población, sin embargo, en la últimas elecciones, a pesar de conseguir un resultado récord para un partido de esas características en Francia en la primera vuelta (34\% de los votos), en la segunda vuelta fue derrotada por Emmanuel Macron, líder del recién creado Partido Liberal en Francia, que demostró que el agotamiento de la población con la élite y los partidos tradicionales, que se ha producido en un número importante de Estados de la UE, se puede vencer sin acudir a los radicalismos nacionalistas.

5 Se impuso con tan solo un punto y medio porcentual al anterior presidente en las últimas elecciones de 2015.

6 Esta derecha radical danesa está representada por el Partido Popular Danés y en las últimas elecciones de 2015 lograron ser la segunda fuerza parlamentaria, garantizando la mayoría absoluta a la derecha moderada del Partido Liberal.

El ukip (Partido de la Independencia Británica), tras lograr el 12,6\% de los votos en las penúltimas elecciones, en las de 2017 apenas consiguió el 2\%, por lo que se puede apreciar un rechazo al partido que apoyó y llevó al Reino Unido al Brexit.

8 En el Parlamento europeo los cupos están asignados por países, sin embargo, la representación y la situación física en el hemiciclo se realiza por partidos políticos. Al no tener un sistema electoral común y una circunscripción única para estas elecciones, los partidos nacionalistas pueden lograr acceder a él aunque sea con algún eurodiputado. Así, el Frente Nacional está presente en el PE con 21 escaños, la Liga Norte de Italia tiene 5, el Partido de la Libertad holandés, 4, etc. 
a la UE el bajo crecimiento económico de sus países, también la entrada masiva de refugiados e inmigrantes, aunque es cada Estado miembro el responsable de guardar sus fronteras, por lo que no son capaces de ver a la UE como una solución sino como la causa de todos sus males. Por esto es que han intentado socavar la UE, desde el retiro de la misma, como lo hizo el Reino Unido, como el incumplimiento de numerosas directivas por parte de los demás Estados nombrados más arriba. Por tanto, cabría preguntarse si estos movimientos nacionalistas serán capaces de impedir que la integración europea siga su curso hacia cada vez más Europa. Se va a demostrar que la UE posee las herramientas necesarias para que estos movimientos no puedan perjudicar la integración, además se demostrará que, desde que surgieron, en la mayoría de las cuestiones se ha avanzado hacia una mayor integración y no, como ellos pretenden, hacia una renacionalización de Europa.

En primer lugar, se va a definir el problema, es decir, qué tipos de nacionalismos han surgido en la UE y cómo cada uno de ellos considera que la UE es el problema y no la solución a los retos que esta enfrenta. En segundo lugar, se va a realizar un estudio de las diferentes herramientas que poseen las instituciones europeas para que estos movimientos no afecten a las dinámicas de integración de la Unión. Para terminar, analizaremos cómo la UE ha seguido avanzando a pesar de que estos movimientos nacionalistas han hecho todo lo posible para que esto no ocurra.

\section{¿CUÁL ES EL RETO AL QUE SE ENFRENTA LA UNIÓN EUROPEA?}

Para analizar si la UE es o no capaz de afrontar el reto del surgimiento de los nacionalismos en su esfera de acción, es necesario definir ese reto al que se enfrenta. No se va a intentar profundizar demasiado en la definición de nacionalismo en este artículo porque, en primer lugar, no es el objeto del mismo y, en segundo lugar, hay mucha literatura sobre el tema y poco más se aportaría desde este escrito. Nos quedaremos con la afirmación de Weber que sostiene que no se puede definir algo tan complejo como la nación de una manera unívoca. Sin embargo, esta dificultad se puede entender como una característica propia de la nación, ya que

distintos grupos humanos tienen visiones distintas sobre qué es una nación "en general” y allí radica justamente una de las grandes virtudes de la combinación entre nación y la modernidad: su capacidad de crear una apariencia de homogeneidad justamente a partir del hecho de que sus prácticas y sus representaciones públicas son extremadamente diversas (Chernilo, 2005, p. 312).

Para definir el nacionalismo en este contexto de la integración europea, se va a acudir solo a las características del mismo que van a ofrecernos los argumentos para demostrar que la UE y su proceso de integración no está en peligro.

El nacionalismo, para lo que atañe a este escrito, se puede definir, en palabras de Breuilly (2005, pp. 16-17) como "un movimiento 
político para el logro y mantenimiento de autonomía, unidad e identidad en nombre de un pueblo, algunos de cuyos miembros consideran que constituye una nación real o potencial". Dentro de esta definición ya se puede ir identificando a alguno de esos movimientos que están presentes en Europa, como podría ser el Frente Nacional de Marine Le Pen o el UKIP liderado en un principio, $y$ hasta que se decidió la salida de la UE, por Nigel Farage.

Esta definición sirve para ir situando a los movimientos nacionalistas europeos, sin embargo, cabe hacer clasificaciones que son necesarias para entender el fenómeno en Europa. Una de estas clasificaciones, a la hora de definir el nacionalismo, es aquella que distingue entre el nacionalismo cívico y el nacionalismo étnico. El primero se caracteriza por ser inclusivo y voluntario, poniendo énfasis en el territorio histórico, en la comunidad política legal y en la cultura cívica. El segundo tipo de nacionalismo, el étnico, se caracteriza por ser exclusivo y orgánico, definido por una comunidad basada en el lugar de nacimiento y en una cultura nativa (Khon, 1946; Smith, 1991; Zimmer, 2003). Con respecto al primer tipo, el cívico, estaría, por ejemplo el UKIP, mientras que los partidos que se identifican más con el étnico en Europa serían, por ejemplo, el Frente Nacional de Francia, el Partido por la Libertad en Holanda o el Partido Auténticos Finlandeses, todos ellos obtuvieron escaños en el Parlamento europeo en las últimas elecciones.

Otra de las características del nacionalismo que sirve para definirlo es que está basado en la distinción del "nosotros" y el "ellos". Aunque en la anterior clasificación se ha observado que se puede hablar de nacionalismo inclusivo y exclusivo, cualquiera de los dos está basado en la diferencia con el otro. En el caso de la UE la amenaza es doble, por un lado se encuentra la amenaza de la pérdida de soberanía de los Estados en favor de la UE ("ellos" en este caso serían las instituciones europeas y los demás Estados miembros o sus nacionales) y, por otro lado, los inmigrantes y los refugiados que llegan a Europa ("ellos" en este caso serían los no nacidos en su territorio y que tienen diferente cultura, religión, idioma, costumbres, etc.).

Por último, cabe destacar una característica de los nacionalismos que, al final, les ofrece una desventaja muy grave a la hora de actuar como movimiento político. El nacionalismo en sí no es una ideología, sino que los diferentes movimientos nacionalistas que se identifican, en este caso en Europa, tienen distinta ideología y solo dos puntos de encuentro, existen movimientos nacionalistas tanto de extrema derecha (Frente Nacional, Jobbik) y movimientos nacionalistas de extrema izquierda (Syriza). Estos partidos, como se decía, solo tienen dos puntos en común, el euroescepticismo y su posición contraria hacia el sistema (nacional o supranacional).

Como se decía antes, se podría profundizar mucho más en el concepto de nacionalismo, sin embargo, para efectos de este escrito, con las características enunciadas, y que se identifican en los movimientos nacionalistas dentro de la UE, es suficiente para abordar la 
cuestión que nos planteamos al principio sobre si la UE es la solución al fin o, por lo menos, a la no injerencia de manera significativa, de estos movimientos políticos en las esferas nacionales y supranacionales (cada vez más difíciles de distinguir).

\section{MECANISMOS EUROPEOS PARA COMBATIR LOS NACIONALISMOS}

Como se ha podido observar de lo antes escrito, solo se va a hacer referencia a lo que el autor considera un reto para la UE, los nacionalismos de carácter estatal, es decir, aquellos que identifican la nación con el Estado actual. No se va a prestar atención en este artículo a los nacionalismos que se han creado dentro de cada Estado y que amenazan la soberanía del mismo sobre territorios del mismo Estado, como el nacionalismo catalán en el caso de España o el escocés en el caso del Reino Unido.

Esto da pie a comenzar por un principio en el que se basa la UE para actuar, el principio de autonomía. Este principio está fundado en que la Unión no va a interferir ni va a imponer a ninguno de sus miembros una forma de Estado, es decir, cada Estado es libre de adoptar una forma acorde con su historia o con su evolución política, por esto en la UE podemos encontrar monarquías con jefes de Estado sin apenas funciones en los que los representantes efectivos son los jefes de gobierno (España, Bélgica, Holanda, Dinamarca, entre otros) o repúblicas, conformadas por jefes de Estado y primeros ministros o presidentes (Francia, Alemania, Austria). Tampoco la UE se pue- de inmiscuir en el hecho de que los Estados adopten sistemas centralizados o federales, por lo que también se encuentran en la Unión países como Francia, altamente centralizados, o como Alemania, Austria o España que son considerados Estados federados.

Teniendo en cuenta lo anterior, en lo que sí puede intervenir la UE es en el caso de que alguno de los Estados no cumpla con los criterios necesarios para formar parte de ella. Estos son los llamados criterios de Copenhague (1993) que definen las características que ha de tener un Estado para ser miembro de la Unión Europea. Los tres criterios son:

- Conformar un Estado de derecho en el que se respeten los derechos humanos y las libertades fundamentales.

- Establecer una economía libre de mercado.

- Acoger el acervo comunitario en la legislación interna de cada Estado.

Estos criterios fueron impuestos en 1993, tras la caída del Muro de Berlín y previendo la entrada de los países que habían sido satélites de la Unión Soviética.

La amenaza que suponen los partidos nacionalistas en donde han llegado a gobernar (Hungría, Polonia) o donde han formado coaliciones para ser parte de los gobiernos (Holanda, Austria, Dinamarca) es que estos provoquen el no cumplimiento de los criterios que más arriba se nombraron. Así, en Hungría se ha producido una persecución por parte del gobierno del presidente Viktor Orán a medios de comunicación, universidades y organizacio- 
nes no gubernamentales, violando el criterio de respetar el Estado de derecho y las libertades fundamentales.

Cuando ocurren este tipo de actos dentro de los Estados, la Comisión Europea puede actuar de oficio o por demanda de algún Estado o institución nacional para investigar si realmente se está incumpliendo con dicho criterio. En Austria, por ejemplo, cuando el Partido Liberal Austriaco (de extrema derecha) conformó gobierno con el centro derecha, la Comisión Europea envió a dicho país un grupo de "sabios" que realizó un informe valorando si se estaba violando, de alguna manera, el Estado de derecho.

En el caso de que se advierta que las medidas nacionales van en contra de alguno de los principios, el artículo 47 del Tratado de Funcionamiento prevé unos procedimientos para que las instituciones europeas actúen en consecuencia. En primer lugar, se advierte al gobierno de turno que su actuación podría ir en contra de los principios de la UE. Si las advertencias no son suficientes, la Comisión Europea puede actuar haciendo que los presupuestos asignados, tanto por los Fondos de Cohesión como por los Fondos Estructurales, no lleguen al Estado afectado. Por último, si las dos medidas anteriores no son suficientes y el Estado en cuestión continua con el incumplimiento, se puede llegar incluso a retirarle temporalmente el voto en el Consejo, lo cual dejaría a dicho Estado fuera de la toma de decisiones de la UE.

En la Unión Europea existe una división de las instituciones que tiene que ver con los intereses que representa cada una de ellas. En esta medida es que pueden actuar. Así, la Comisión Europea representa el interés general de la UE, por lo que todas sus actuaciones irán encaminadas hacia ese objetivo. El Parlamento europeo representa los intereses de los ciudadanos europeos, por lo que esta institución, en la práctica, no puede tomar medidas contra los gobiernos de los Estados ya que, estos, a su vez representan a los ciudadanos de dicho Estado, que se supone que tiene órganos de control democrático. El Consejo, tanto el de la Unión como el Europeo, representa los intereses particulares de cada uno de los Estados, por lo que también se encuentra muy atado porque los partidos nacionalistas que gobiernan se aprovechan del mismo para defender sus intereses (que, en principio, son tan legítimos como los de los demás). Una de las medidas que se está pensando en adoptar en el Consejo de la Unión es impedir que haya cuestiones que necesiten la unanimidad como, por ejemplo, la entrada de nuevos miembros. Esto provocará que los partidos de carácter nacionalista que gobiernen y que, por definición son euroescépticos, no impidan que el proceso de integración se vea frenado.

Hasta aquí llegan los mecanismos legales de la UE para impedir que los gobiernos con partidos nacionalistas al frente puedan culminar sus propósitos dentro de cada uno de los Estados. Son mecanismos que nunca se han utilizado, que son lentos y que tienen un carácter controvertido porque no todos los Estados están de acuerdo con que la UE se tenga que inmiscuir en ciertas cuestiones. Además, son 
mecanismos que requieren de la unanimidad de los demás Estados y esta es difícil de conseguir en una Europa de 28 Estados.

Sin embargo, la dinámica del proceso de integración y los compromisos ya adquiridos por los miembros, son mucho más eficaces a la hora de echar por tierra las promesas que este tipo de partidos hacen a sus ciudadanos para llegar al poder. El caso más reciente y más significativo en este sentido puede ser el del partido de izquierda radical de Grecia Syriza. Este partido llegó al poder en plena crisis económica prometiendo a sus ciudadanos que no se iban a someter a las medidas de austeridad que desde la UE se promulgaron para que los diferentes Estados afectados por la crisis la combatieran. El primer ministro griego Alexis Tsipras, incluso llegó a decir que podría abandonar el euro si no se respetaba lo que él pretendía hacer en su país, sin embargo, las negociaciones con el resto de Estados y con el Banco Central Europeo no le dieron margen de maniobra ya que su situación de deuda era extrema. Realizó un referéndum para que sus ciudadanos votaran si aceptaba o no las condiciones que ponía la UE para acceder a los rescates previstos, el resultado fue negativo, en el sentido que sus ciudadanos decidieron que no acogiera dichas condiciones. Fue a negociarlas a Bruselas y no tuvo más remedio que acogerlas para acceder a los rescates, produciéndose así la dimisión de su ministro de economía (Yanis Varoufakis) y también su propia dimisión ya que no había seguido el resultado de lo que habían dicho sus ciudadanos, que al final, lo reeligieron tras convocar a unas elecciones provocadas por su dimisión.

La regla de la mayoría es la norma general en la toma de decisiones en la UE, por lo que los griegos, en este caso, no tenían margen de negociación, pues otros países se habían visto afectados de manera similar por la crisis y, por su carácter europeísta o convencidos que dicha austeridad era la solución, ya habían tomado las medidas que ahora se le imponían a Grecia. Fueron, por ejemplo, los casos de Portugal e Irlanda, que además fueron exitosos en cuanto a la salida de la crisis y la vuelta a un crecimiento sostenido de su economía.

Por lo tanto, ese nacionalismo, esa defensa de la soberanía, ese exacerbamiento de lo particular en un entorno sin fronteras, compartiendo una moneda, un mercado común y en medio de una crisis, resulta más un llamamiento al sentimiento nacional, a la emoción, para llegar a gobernar y tener el poder, que a una estrategia de solución de los problemas internos que cada vez lo son menos, y una visión de la realidad muy distorsionada en la que no se comprende muy bien que la solución está en Europa y no en cada Estado.

Para ejemplificar y dar evidencia de lo que suponen los mecanismos de la UE para combatir movimientos nacionalistas, cabría mencionar también el caso del Reino Unido y el famoso Brexit. Sin embargo, por cuestiones de extensión de este artículo, simplemente se hará referencia a que la misma UE, y a propuesta del Reino Unido, por primera vez en los tratados se tenía previsto que un Estado decidiera abandonar el proceso de integración, por lo que la manera como se puede combatir esa posibilidad es posicionándose firmemente en las negociaciones de la salida de dicho Estado. Esto es lo que ha ocurrido con el Reino Unido, los demás Estados han hecho un frente común dándole el poder de negociación a la 
Comisión Europea (recordemos que es la que representa el interés general de la UE). Además, en contra de los pronósticos de que el caso del Reino Unido podría resultar en un efecto dominó, ni los países gobernados por partidos nacionalistas (Polonia, Hungría) han decidido seguir el mismo camino que los nacionalistas ingleses provocaron.

La verdad es que la UE, de manera legal, tiene pocos instrumentos para combatir a los movimientos nacionalistas que surgen en los Estados y que tienen un carácter euroescéptico. Sin embargo, como se verá a continuación, el peso mismo del proceso y su cada vez más incidencia en los ciudadanos europeos, además de las contradicciones y paradojas de estos movimientos, hace que el nacionalismo no sea una amenaza real al proceso de integración y que cada vez se sea más consciente que la solución supera los ámbitos nacionales.

\section{EL PESO DE LA UE Y LAS PARADOJAS NACIONALISTAS}

Para este último apartado en el que se va a argumentar y demostrar la hipótesis propuesta, se va a hacer referencia a las definiciones y clasificaciones que se hicieron más arriba y que sirvieron de marco teórico o conceptual del artículo.

En un primer lugar, se había distinguido entre un nacionalismo cívico (inclusivo) y étnico (exclusivo), ambos presentes en los diferentes Estados miembro de la UE. Como se decía, más mecanismos legales y tangibles de la Unión no existen, sin embargo, sí existe lo que se ha denominado la identidad europea.
Hay una cantidad importante de literatura acerca de la identidad europea (Checkel $\&$ Katzenstein), sin embargo, me voy a basar para este primer argumento en la distinción que hacen Wodak y Boukala de lo que supone la identidad europea. Distinguen entre tres tipos que se dan en la Unión: una identidad europea como una identidad nacional, una identidad europea como una identidad posnacional y una identidad supranacional.

Los nacionales de los países de la UE se sienten cada vez más europeos. Más arriba, se hablaba del nacionalismo inclusivo, Risse (2010) lo relaciona con este sentimiento de primero sentirse nacional de un país, pero también perteneciente a Europa. En contra de lo que se denomina nacionalismo exclusivo, a través del cual no puede haber un sentimiento europeo, sino que los ciudadanos se sienten pertenecientes a su país y ya. Las cifras del Eurobarómetro de 2017 muestran que el nacionalismo inclusivo es el más predominante en la UE, puesto que un 70\% de los ciudadanos de la UE se identifican con ella. Hay variaciones en los países, del más alto que es Luxemburgo (90\%) y España (88\%) hasta el 52\% de los griegos o el 55\% del Reino Unido (como sabemos ya con un pie fuera de la UE). Cabe resaltar que la crisis económica tiene parte de culpa de los resultados griegos, sin embargo, un país como España que sufrió también la crisis de una manera más profunda que otros de la UE, sigue reflejando un resultado muy positivo en cuanto a su identificación con la UE. Por esto es que el fenómeno del nacionalismo y sus constantes resurgimientos tienen múltiples causas y es muy difícil de estudiar. 
España ha sido uno de los países en que no ha surgido un fenómeno nacionalista a nivel del Estado, aunque sí surgió a nivel local, pero las causas de este tipo de nacionalismos son externas a la UE y el nacionalismo catalán no tiene ese sentimiento antieuropeo que caracteriza a los nombrados y estudiados en este artículo.

Los otros dos tipos de identidad que se nombraban tienen que ver con la necesidad de crear una identidad política de la UE con la que los ciudadanos se puedan identificar, Habermas (2001, p. 5) habla de "una asociación de Estados soberanos que cederían su soberanía en áreas muy restringidas y en varios grados, una asociación que no buscaría tener poder coercitivo para actuar directamente en los individuos del modo que lo hacen los Estados nación”.

En cuanto a lo que se refiere a la identidad supranacional, Smith (1995) explica que no se ha tenido éxito en crear una identidad europea sobre las nacionales porque ha sido un proceso inventado por las élites que no ha llegado al ciudadano y en el que se toman decisiones de manera poco transparente para el mismo. Este proceso, por tanto, debería llegar a los ciudadanos, y de hecho está llegando porque muchos se sienten identificados con los logros de la UE por cuanto se ven directamente afectados. Así, según el Eurobarómetro de 2017, lo más positivo que los ciudadanos encuentran de la UE es el libre movimiento de los factores productivos, la paz entre los Estados de la UE, el intercambio entre estudiantes y el euro. Todos estos logros, poco a poco se van identificando con la Unión y cada vez menos con políticas de los Estados hacia sus ciudadanos.

Como decíamos antes, el nacionalismo se fomenta con la diferenciación del "nosotros" y el "ellos". Para los partidos nacionalistas que han surgido en los Estados europeos, "ellos" son los partidos políticos tradicionales que han sido esa élite de la que hablábamos, son las instituciones europeas. Son ellos los causantes de la crisis por creer en la UE y en el euro, son ellos los responsables de que sus fronteras no sean fuertes y sean permeables. Volviendo al Eurobarómetro, en este caso sí hay coincidencia del surgimiento de estos partidos nacionalistas con las principales cuestiones que preocupan a los europeos, las dos primeras son el desempleo y las migraciones. Estos partidos, como la definición de nacionalismo indicaba, se aprovechan de un sentimiento negativo de la gente frente a una situación que en algunos países como Grecia, se ha vuelto estructural, y estos partidos ofrecen la falsa esperanza de que fuera de la UE les iría mejor, sin contar en ningún momento qué pasaría si en realidad salirse de la UE fuera una opción. Los ingleses lo van a saber, se supone, el próximo año en marzo, cuando ya se ha tenido que terminar la negociación para un período de transición de dos años, sin embargo, no se ha podido llegar a un acuerdo puesto que la Unión no va a dejar que los Estados que quieran salirse se sigan beneficiando de todo lo que supone estar dentro.

Las primeras comunidades europeas y la posterior Unión Europea se crearon sin un "nosotros" y sin ningún "ellos", esta Unión es fruto de una identificación en contra de lo que había sido Europa durante toda la historia, una Europa de guerras, una Europa en la que la guerra era un instrumento para dirimir conflictos y que terminó con el mayor desastre que la humanidad haya conocido, la segunda guerra mundial que se llevó a 55 millones de 
personas. Esta Europa no en este caso "ellos" y en la actualidad, sería un retroceso en el proceso de integración que los Estados volvieran a tomar medidas que provocaran tener situaciones similares a lo que fue aquella Europa, más cuando se han desmantelado todas las fronteras internas de Europa y cuando se comparten muchas más cosas de las que marcan una diferencia entre ellos, incluyendo los retos a los que se enfrentan.

En este caso, y a falta de mayor evidencia, cabe destacar que los ciudadanos europeos, según el Eurobarómetro de 2017, se sienten en su mayoría optimistas con el futuro de la UE, incluso se muestra que los ciudadanos tienen más confianza en la gobernanza de la Unión que en sus propios gobiernos o partidos nacionales. Una muestra más de que el nacionalismo no supone una amenaza para el proceso de integración europeo, sino que, por el contrario, este está calando cada vez más profundo en la conciencia de los ciudadanos que ven cada vez a la UE como la solución y no el problema.

Para acabar con la argumentación y seguir mostrando que los nacionalismos esporádicos que han surgido a lo largo y ancho del continente europeo no suponen una amenaza para el proceso de integración, cabe indicar que dichos movimientos han podido ser relevantes a nivel nacional (Reino Unido, Hungría, Polonia o Grecia), sin embargo, como representación en el Parlamento europeo, son minoría, y una minoría dividida, ya que pertenecen a ideologías muy distantes o, en otros casos, simplemente no quieren ser identificados con otros movimientos nacionalistas que tienen programas mucho más radicales, ya que esto les haría perder el apoyo necesario para estar presentes en las instituciones europeas.

En las últimas elecciones al Parlamento europeo, varios de estos partidos nacionalistas y antieuropeos llegaron a tener eurodiputados, la cantidad suficiente incluso para crear un grupo parlamentario como ya existe el del Partido Popular Europeo o el Socialista o los liberales. Sin embargo, por las causas que se acaban de nombrar, no pudieron conformar dicho grupo, lo cual ha hecho que pierdan mucha relevancia aunque sigan presentes. De hecho, incluso el partido Frente Nacional de Marine Le Pen, está siendo investigado por corrupción de los fondos que son asignados a cada partido por poseer representación en el Parlamento europeo.

Esta desunión de los nacionalistas hará casi imposible que se puedan poner de acuerdo a nivel europeo para proponer algo diferente al proceso de integración que se continúa llevando a cabo después de sesenta años de éxitos. Quizás lo único que se pueda hacer es desestabilizar a los Estados a nivel interno y que esto, de manera indirecta, afecte el proceso. No obstante, en la medida que se vayan cambiando los procesos y la mayoría cualificada sea la regla sin excepción en la toma de decisiones en la Unión, este problema indirecto podrá ir desapareciendo. Una solución también sería la de poner una circunscripción única europea para las elecciones al Parlamento, que hoy en día también deciden quién será el presidente de la Comisión Europea. Por ahora esto no es viable, como tampoco lo es la celebración de un referéndum a nivel europeo, pero seguro que será una herramienta, que en caso de ser utilizada, 
facilitará los procesos en la UE y hará más difícil el surgimiento de movimientos que ya no solo se tendrían que legitimar a nivel nacional sino que también tendrán que hacerlo a nivel europeo. El único peligro sería la creación de una especie de nacionalismo europeo, que en todo caso sería un nacionalismo inclusivo, en el que cabrían todos los ciudadanos europeos y en el que no cabría la exclusión por lugar de nacimiento, algo que está regulado y que es un derecho en la actualidad en la UE, el derecho a la no discriminación.

\section{CONCLUSIÓN}

Se partía en este escrito de la hipótesis de que los movimientos nacionalistas que habían surgido en los diferentes Estados de la UE no suponían una amenaza para el proceso de integración europeo. Se ha demostrado que la UE y sus instituciones tienen mecanismos para contrarrestar las posibles infracciones que en nombre del nacionalismo puedan realizar estos movimientos, aunque hasta ahora no se hayan utilizado más que las advertencias y los grupos de investigación para la inspección en cada uno de los Estados en donde ha habido sospecha de que esto pudiera ocurrir.

Por otra parte, se ha demostrado que la identidad europea ya es algo que está ahí y que tiene su peso. Los ciudadanos europeos ya se han acostumbrado a vivir con una serie de elementos que han sido proporcionados por el proceso de integración y que, echarlos hacia atrás supondría un retroceso en dicho proceso y un coste para los Estados en general y para los ciudadanos, como personas, trabajadores y como consumidores, en particular. Ya no sirve echar mano de los sentimientos nacionalistas para inventar soluciones mágicas a los problemas que, en la actualidad, no lo son de un solo Estado, sino de todos. Si pudiéramos poner un antónimo al nacionalismo, este sería el cosmopolitismo, el proceso de globalización, que tiene su mayor expresión en la Unión Europea, es precisamente eso, una expresión de cosmopolitismo que ha creado una etapa, la más larga de la historia, de paz y progreso en Europa, algo que ni los ciudadanos europeos ni los diferentes gobiernos están dispuestos a asumir que se acabe.

Esto no quiere decir que no surjan nuevas preguntas y nuevos retos que se han de superar de manera original en el escenario europeo. Como siempre se dice, la UE es un proceso inacabado y, al parecer, siempre lo será, esto quiere decir que cada vez hay que ir construyendo bajo los principios que concibieron el proceso, la paz y la solidaridad, algo que los partidos nacionalistas no están dispuestos a ofrecer. De esta manera, existen muchas cuestiones por resolver y la UE y los diferentes gobiernos de los Estados miembros se tienen que poner de acuerdo para solucionarlas. ¿Es el problema del desempleo algo que se tenga que resolver a nivel nacional o la Unión puede hacer algo al respecto que beneficie de manera directa a sus ciudadanos? ¿Las migraciones necesitan de una política europea que vaya más allá de la instauración de unos formularios Schengen unificados? ${ }_{¿} \mathrm{H}$ a de ser una política europea la que decida quién entra y quién no en Europa o los Estados deben mantener la soberanía en esas cuestiones? ¿Es posible solucionar una 
crisis a nivel nacional con el grado de interdependencia que supone formar parte de la UE y tener una moneda en común con 19 países?

Todas estas cuestiones se plantean constantemente en la UE, y su respuesta nos dará la clave de algo que los partidos nacionalistas, que han querido llegar al poder en los diferentes Estados, se niegan a admitir, que la Unión Europea no es el problema sino la solución.

\section{BIBLIOGRAFÍA}

Álvarez Peralta, I.; Luengo Escalonilla, F. y Uxó González, J. (2013). Fracturas y crisis en Europa, en Papeles de Relaciones Ecosociales y Cambio Global, No. 1242013/2014, pp. 189-199, Madrid: Clave Intelectual y Eudeba.

Álvarez, M. V. (2012). El euroescepticismo en una Unión Europea en crisis: ¿viejos fenómenos en nuevos órdenes?, en Revista de Integración y Cooperación Internacional, No. 13, octubre/diciembre. En: http:// rephip.unr.edu.ar/bitstream/handle/2133/5684/ Articulo\%201\%20-\%20Revista\%20Nro.\%20 13.pdf? sequence $=3 \&$ isAllowed $=y$

Brumat, L. (2013). Políticas migratorias, construcciones de identidad europea y el inmigrante como una "amenaza". Un análisis de la Directiva de Retorno, en Diversidad, junio, No. 6, año 4. En: http:// www.diversidadcultural.net/articulos/nro006/0601-leiza-brumat.pdf_ISSN 2250-5792.

Checkel, J. T. y Katzenstein, P. J. (2009). European Identity, Cambridge: Cambridge University Press, ISBN 978-0-521-88301-6.

Chernilo, D. (2013). Las relaciones entre nacionalismo y cosmopolitismo, en Papers Revista de Sociología, 100/3, 303-324, Loughborough University. En: http://dx.doi.org/10.5565/rev/papers.2142
De Ayala, J. E. (2010). Crece el nacionalismo, un nuevo problema de la Unión Europea, en Estudios de Politica Exterior S.A., vol. 24, No. 136 (jul./ ago.), pp. 14-20.

De Blas Guerrero, A. (1984). Nacionalismo e ideologias politicas contemporáneas, Madrid: Espasa Universitaria, Espasa Calpe.

De Regoyos, J. (2011). Bélgica: El laboratorio nacionalista de Europa, en Cuadernos de Pensamiento Político, No. 13, julio/sept., pp. 101-133, Fundación para el Análisis y los Estudios Sociales, Faes. Eurobarómetro. (2017). En: file://C:/Users/MMG/ Downloads/eb88_first_en.pdf

Fligstein, N.; Polyakova, A. y Sandholtz, W. (2012). European Integration, Nationalism and European Identity, en Journal of Common Market Studies, vol. 50, No, S1, 106-122. Blackwell Publishing.

Forner, S. y Senante, H. C. (2014). La crisis de Europa y el ascenso del populismo, en Cuadernos de Pensamiento Politico, No. 43, julio/sept. 51- 67, Fundación para el Análisis y los Estudios Sociales, Faes. Gagnon, A.; Lecours, A. y Nootens, G. (Ed.). (2011). Contemporary Majority Natinalism Quebec, Canadá: McGill-Queen's University Press, Universidad de Quebec. ISBN 978-0-7735-3825-2.

Gayo Cal, M. (2010). ¿Es posible escapar del nacionalismo?, en Papers Revista de Sociología, 95/2, 341 - 361. Santiago de Chile: Universidad Diego Portales. En: http://dx.doi.org/10.5565/rev/ papers/v95n2.68

Guibernau, M. (1996). Los nacionalismos, Barcelona: Polity Press, Editorial Ariel, ISBN 84-344-1808-8.

Halikiopoulou, D.; Mock, S. y Vasilopoulou, S. (2012). The civic zeitgeist: nationalism and liberal values in the European radical right, en Nations and $N a$ tionalism, vol. 19, Issue 1, 107-127. En: https:// doi.org/10.1111/j.1469-8129.2012.00550.x 
Halikiopoulou, D.; Nanou, K. y Vasilopoulou, S. (2012). The paradox of nationalism: The common denominator of radical right and radical left euroscepticism, en European Journal of Political Research.

Khon, H. (1944). The idea of Nationalism, London: Macmillan.

McKim, R. y McMahan, J. (1997). The Morality of Nationalism, Oxford: Oxford University Press, Inc. ISBN (vol. 1) 84-7432-891-8.

Sanahuja, J. A. (2012-2013). Las cuatro crisis de la Unión Europea, en Anuario CEIPAZ, No. 5, Fundación Dialnet, Universidad de La Rioja, pp. 51-84, ISSN 2174-3665.
Smith, A. D. (1995). Nations and Nationalism in the Global Era, Cambridge: Polity Press, ISBN 0-74561019-6.

Wodak, R. y Boukala, S. (2015). European identities and the revival of nationalism in the European Union, en Journal of Lenguage and Politics, pp. 87-109, John Benjamins Publishing Company ISSN 1569-2159 / E- ISSN 1569-9862. 